\title{
Dissociation of dorsal hippocampal regional activation under the influence of stress in freely behaving rats
}

\author{
Johannes Passecker ${ }^{1}{ }^{*}$, Vincent Hok $^{1}$, Andrea Della-Chiesa ${ }^{1}$, Ehsan Chah ${ }^{1,2}$ and Shane M. O'Mara ${ }^{1}$ \\ ${ }^{1}$ Trinity College Institute of Neuroscience, Trinity College Dublin, Dublin, Republic of Ireland \\ ${ }^{2}$ Trinity Centre for Bioengineering, Trinity College Dublin, Dublin, Republic of Ireland
}

\section{Edited by:}

Jeansok J. Kim, University of Washington, USA

\section{Reviewed by:}

Min W. Jung, Ajou University School of Medicine, South Korea

David M. Smith, Cornell University, USA

\section{*Correspondence:}

Johannes Passecker, Trinity College Institute of Neuroscience, Trinity College Dublin, Dublin 2, Republic of Ireland.

e-mail: passeckj@tcd.ie

\begin{abstract}
Stress has deleterious effects on brain, body, and behavior in humans and animals alike. The present work investigated how 30-min acute photic stress exposure impacts on spatial information processing in the main sub-regions of the dorsal hippocampal formation [CA1, CA3, and dentate gyrus (DG)], a brain structure prominently implicated in memory and spatial representation. Recordings were performed from spatially tuned hippocampal and DG cells in rats while animals foraged in a square arena for food. The stress procedure induced a decrease in firing frequencies in CA 1 and CA3 place cells while sparing locational characteristics. In contrast to the CA1-CA3 network, acute stress failed to induce major changes in the DG neuronal population. These data demonstrate a clear dissociation of the effects of stress on the main hippocampal sub-regions. Our findings further support the notion of decreased hippocampal excitability arising from behavioral stress in areas CA1 and $\mathrm{CA}$, but not in $\mathrm{DG}$.
\end{abstract}

Keywords: stress, freely moving, place cells, hippocampus, CA1, CA3, dentate gyrus

\section{INTRODUCTION}

The hippocampus has received a huge amount of attention due to its particular involvement in several memory processes such as the episodic and spatial memory. In addition, it is widely accepted that the hippocampus plays a critical role in the stress response. The amygdala, a central stress regulating structure (LeDoux, 1994; Cahill and McGaugh, 1998; Roozendaal et al., 1998; McGaugh, 2000; Kim et al., 2005), projects directly (Cahill and McGaugh, 1998) and indirectly (through the entorhinal cortex, McDonald and Mascagni, 1997) to the hippocampus. The hippocampus is also subject to mainly mineralocorticoid receptor (MRs) and glucocorticoid receptor (GRs) activation as part of the HPA axis activation (de Kloet et al., 2005). The extent of this regulation appears to be critically dependent in a time- and dose-dependent manner (Schwabe et al., 2010).

Virtually all of the electrophysiological studies on the stress response published so far have focused on in vitro studies or in vivo studies of anesthetized rats (e.g., Karst, 2000; Kole et al., 2001; Alfarez et al., 2002; Karst et al., 2005; Van Gemert and Joels, 2006; Wiegert et al., 2006). It is however, imperative to study neuronal activity during natural behaviors to get a better understanding of how stress shapes hippocampal function and to interpret the underlying deficits and responses observed during behavioral tasks under the influence of stress. Thus we use hippocampal place cells of the three main sub-regions of the hippocampus to study the effects on this crucial network in a period of stress recovery. The hippocampus is a key structure involved in spatial information processing as suggested by the presence of a large population of place cells (O'Keefe and Dostrovsky, 1971; O'Keefe and Nadel, 1978). Such cells are active when the animal occupies specific locations in particular environments (O'Keefe and Conway, 1978; Muller et al., 1987). Place cells are therefore functionally characterized by a location-specific firing which is referred to as a "firing field" or "place field."

Hippocampal place fields in young rats can be stable for months when recorded in a familiar environment (Thompson and Best, 1990); however, small changes in sensory or cognitive inputs can trigger a completely different place field representation (Muller and Kubie, 1987; Leutgeb et al., 2005). These changes of place cell activity constitute a "remapping" and can be of two distinct types (Colgin et al., 2008): (i) "rate remapping" occurs when animals are trained and tested in the same location using different-shaped or colored enclosures; in this case, place fields are stable in location but display rate differences across conditions (Leutgeb et al., 2005); or (ii) "global remapping," when animals are placed in two different testing rooms, whether the enclosure is similar or not (Muller and Kubie, 1987); place fields in this situation change their firing activity along with their spatial location. Hippocampal sub-regions [i.e., CA1, CA3, and dentate gyrus (DG)] appear to be differently affected by the rate remapping process (Leutgeb et al., 2004, 2005, 2007). When taking into account rate and population vectors differences, DG cells activity shows profound changes, far more important than the ones observed in CA3, which is itself more affected by rate remapping than CA1. This process is thought to underlie the mechanism for encoding episodic-like memories about events that have previously occurred within a specific context (Leutgeb et al., 2006; Colgin et al., 2008). Kim et al. (2007) tested the idea of how acute stress affects those remapping processes in the CA1 region. A 2-h audiogenic stress protocol induced higher firing rate changes than during control conditions using a rate remapping protocol in CA1 place cells. Based on those results the authors suggest that acute stress may impair spatial memory processes via rate remapping processes in the hippocampal formation. In order to test whether other sub-regions 
implicated in rate remapping are affected by acute stress and to better understand the underlying processes we recorded CA1, CA3, and DG place cells in a stable environment where a prior stressor had been applied for $30 \mathrm{~min} 1 \mathrm{~h}$ previously. This allows us to investigate in a more sophisticated way hippocampal dynamics during the post-stress phase which is important for learning and memory processes.

\section{MATERIALS AND METHODS ANIMALS}

A total of nine (4-6 months) male Wistar rats (B\&K, UK) weighing between 420 and $530 \mathrm{~g}$ were used. Upon arrival, animals were housed individually and handled by the experimenter daily for a week before being trained in the pellet-chasing task (see below). Rats were food-deprived to $90 \%$ of their ad libitum body weight and kept in a temperature controlled laminar airflow unit and maintained on a 12-h light/dark cycle (lights on from 08:00 to 20:00 hours). Experiments were carried out in strict accordance with regulations laid out by LAST Ireland and were compliant with the European Union directives on animal experimentation (86/609/EEC).

\section{RECORDING AND ANALYSIS}

Detailed descriptions of the surgical protocol and recording techniques can be found elsewhere (Brotons-Mas et al., 2010; Tsanov et al., 2011). Briefly, rats were implanted with tetrodes of either four or eight bundles of platinum-iridium wires (California Fine Wire Ltd., USA) mounted onto small driveable microdrives (Axona Ltd., $\mathrm{UK}$ ) at the following coordinates: $3.5-3.7 \mathrm{~mm}$ posterior to bregma,
2.3-2.8 $\mathrm{mm}$ lateral to the midline and depth varied depending on structure from 1.5 to $3.2 \mathrm{~mm}$ from the dura (see Figure 1; Paxinos and Watson, 1998). Tetrodes were implanted at the identified coordinates and then lowered through the layers. The coordinates were chosen to optimize the passage of the electrodes through all the layers. Based on the daily record of the electrode position and post mortem histological verification each recording could be located along the tetrode trace. Rats were allowed at least 1 week of recovery post-surgery. The 16 -min long recordings took place in a square-shaped arena $(64 \mathrm{~cm} \times 64 \mathrm{~cm} \times 25 \mathrm{~cm})$ located in the center of the room with multiple large visual cues made accessible to allow the animals to orient themselves in the environment. $20 \mathrm{mg}$ food pellets (TestDiet ${ }^{\mathrm{TM}}$, 5TUL formula) were thrown in the arena at random locations ca. every $20 \mathrm{~s}$. During the weeks of recordings animals were allowed $20 \mathrm{~g}$ of food daily. Unit identification was based on several criteria. First, neurons had to be active in both conditions and had to present same waveform characteristics (amplitude, height, and duration) in those conditions. Further, they had to present at least a maximum firing frequency of $1 \mathrm{~Hz}$ and a spatial selectivity of $>3$, in one of the two sessions. Furthermore, to be kept, units had to demonstrate a clean refractory period $(<2 \mathrm{~ms})$ in the autocorrelation. Once well-defined neuronal signals from the respective subareas were isolated and the rats explored the arena sufficiently (rats had to explore at least $90 \%$ of all bins in either session to be included in analysis to allow reliable calculation of spatial characteristics), the stress protocol was initiated. The acute stress protocol consisted of $30 \mathrm{~min}$ photic exposure, using bright light (ca. $120 \mathrm{~cd}$ ) on top of a small bucket in a separate room. This stress protocol has consistently

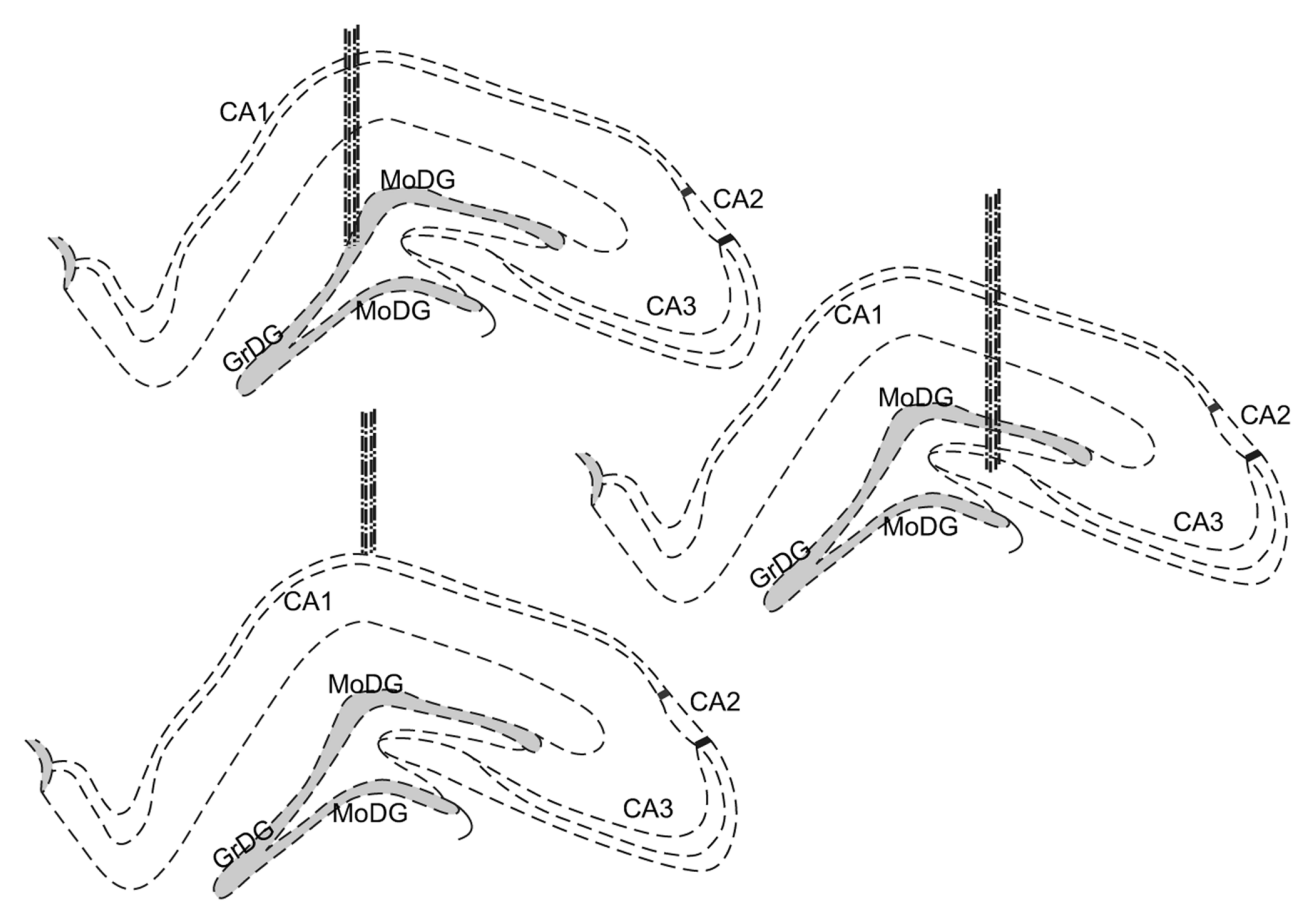

FIGURE 1 | Schematic drawings of the implantation sites; cells were recorded from pyramidal cell layers of the hippocampus, in particular, CA1, CA3, and the granule cell layer of the DG. Dotted lines represent the dense neuronal layers of each structure. The gray shaded areas represent the main granule cell layer of the dentate gyrus. 
induced stress responses and spatial memory deficits (Commins and O'Mara, 2000) as well as drastic performance decreases in spatial memory in Wistar rats (unpublished data). Thus in our lab it has been utilized as a reliable inducer of a systemic behavioral stress response. Rats were then allowed $30 \mathrm{~min}$ of rest in the bucket with lights turned off, before recording in the pelletchasing task for a second time (stress condition). Animals were never stressed twice within a 3-day period in order to decrease the possibility of adaption to the stressor and to prevent chronic stress induction. On average, each animal was exposed to the photic stressor a total of approximately 7.5 times. The spatial specificity (or spatial information content) is expressed in bits per spike and calculated according to Skaggs et al. (1996). Similar to Hollup et al. (2001), place field size is computed as the region of the arena in which the firing rate of the place cell is above $20 \%$ of the maximum firing frequency. A place field was identified if nine neighboring pixels (sharing a side) were above $20 \%$ of the peak firing rate. Place field size was represented in number of pixels. The spatial selectivity of a firing field (ratio of maximal signal to noise) was calculated by dividing the firing rate of the cell in the bin with the maximum average rate by its mean firing over the entire apparatus (Skaggs et al., 1996). Average frequency is the total number of spikes divided by the total recording time and is expressed in $\mathrm{Hz}$. Neuronal units of the second recording were matched based on their spike amplitude, height, and spike duration with the respective units from the control recording. The burst index was calculated by acquiring first the number of spikes within a burst (minimum of two consecutive spikes in $6 \mathrm{~ms}$ ). The number of bursts were then detected, and multiplied by the average number of spikes within a burst. A ratio was then calculated between this number and the overall number of spikes within the recording. Exploration was assessed by comparing the occupancy of bins and the number of visits per bin between the two recording conditions. Data was tested for normality (normal quantile plot). If the test returned a non-normal character of the distribution, a Wilcoxon-signed rank test ( $Z$ values provided in the section Results) was performed, otherwise a parametric paired $t$-test $(t$ values provided) was used.

If no more hippocampal neurons could be recorded or the headstage and the ground wire were irreparably damaged, rats were sacrificed and their brains collected for electrode position verification. Brains were collected and stored in a 4\% PFA solution (Sigma, UK). In short, brains were embedded in paraffin, and the target region was microtome sliced into $20 \mu \mathrm{m}$ sections. Thereafter, brain sections were stained with a standard hematoxylin and eosin staining protocol to allow cellular identification. Tetrode tracks were verified by visual inspection of the experimenter under a standard microscope (Leica, GER) fitted with a $5 \times$ and a $20 \times$ lens (Leica, GER).

\section{RESULTS}

In total, 138 well-defined place cells were recorded in 9 animals, with 99 of those cells fulfilling the matching criteria required to analyze cell activity in both (control and stress) recording conditions. Out of those 99 cells, 34 were recorded from the CA1 region, 18 from CA3, and 47 from DG (main summary of effects in Figure 2, examples presented in Figure 3).

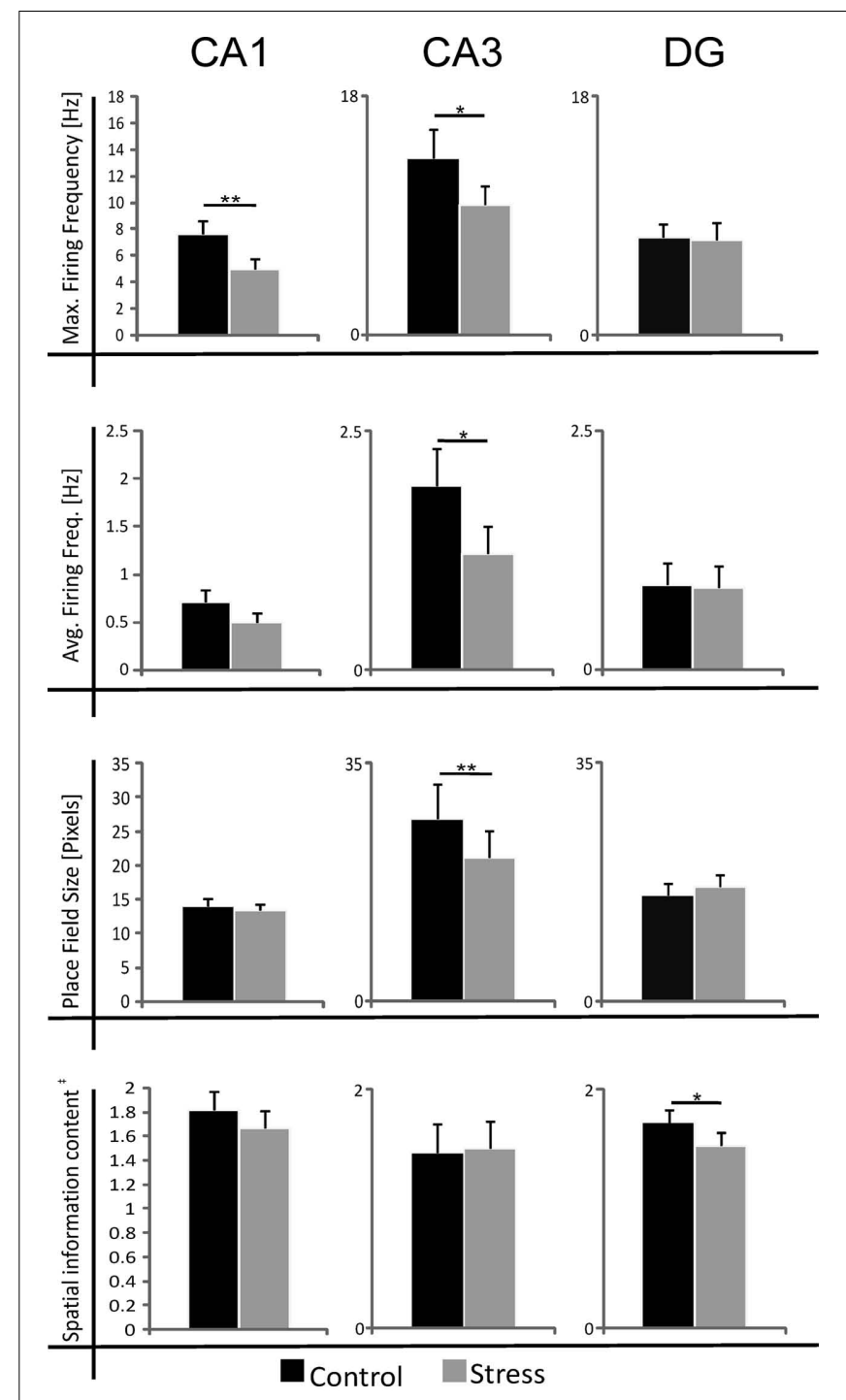

FIGURE 2 | Summary of the effects of acute photic stress on CA1, CA3, and DG cells; CA1 and CA3 cells generally experience a significant decrease in firing activity whereas spatial characteristics are mainly unchanged. In contrast, DG cells do not experience a change in firing rate after stress. Black bars represent mean sample values of control sessions and gray bars represent mean sample values of stress sessions. The size of place fields was defined by the firing frequency above $20 \%$ of the maximum firing frequency. In total 99 cell pairs (control/stress) were recorded. $n_{\mathrm{CA} 1}=34, n_{\mathrm{CA} 3}=18, n_{\mathrm{DG}}=47{ }^{*} p<0.05,{ }^{* *} p<0.01$, ${ }^{\ddagger}$ Spatial information content in bits per spike.

Strikingly, maximum firing rate was significantly decreased (Wilcoxon-signed rank test) for both CA1 $\left(Z_{33}=-2.85\right.$, $p=0.004)$ and CA3 place cells $\left(Z_{17}=-2.04, p=0.041\right)$, but not for DG place cells $\left(Z_{46}=-1.75\right.$, n.s. $)$. Similarly, there was a significant decrease in average firing rate for CA3 units $\left(Z_{17}=-1.96, p=0.049\right)$ and a marginal decrease for CA1 units $\left(Z_{33}=-1.88, p=0.06\right)$, but not significant differences for DG units $\left(Z_{46}=-1.64\right.$, n.s.). Burst analysis of both CA1 and CA3 cells revealed a highly significant decrease in overall burst activity between control and stress sessions. The mean value for the 


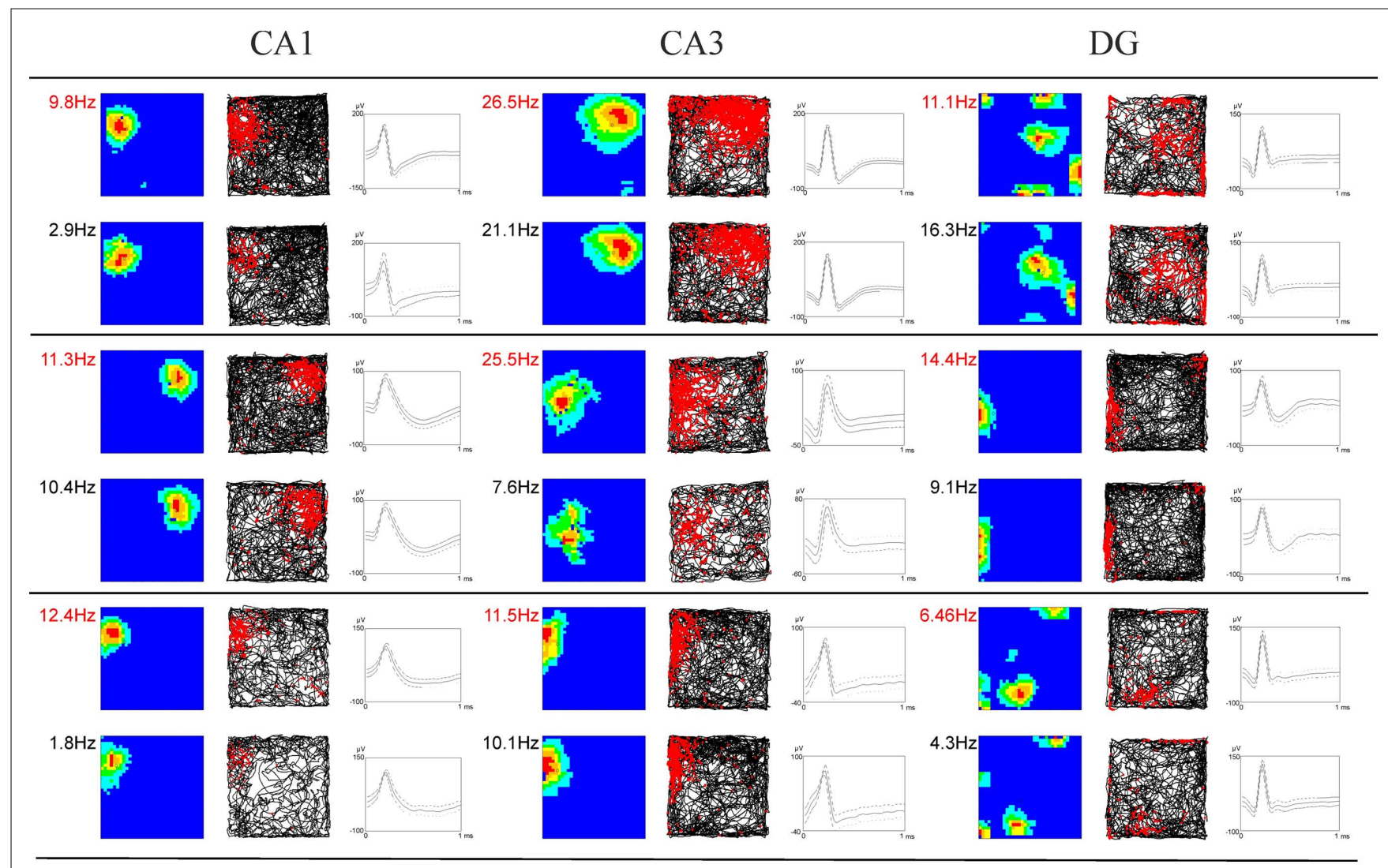

FIGURE 3 | Examples of firing characteristics of hippocampal and subicular neurons. The first column of each subregion depicts the smoothed color coded map showing the firing rate per pixel scaled to the maximum firing rate within the environment at the top left. Dark blue denotes the lowest firing category and red indicates the highest firing category. Color steps are in $20 \%$ of the maximum firing rate. The second column shows the corresponding path trajectories and the corresponding spikes of the neurons in red. The third column depicts the cell signal of the most prominent channel where time is plotted on the $x$-axis and spike amplitude is plotted on the $y$-axis. Two consecutive rows always show the same cell in both the control (firing rate in red) and stress condition (black). burst index within control sessions was $0.224(\mathrm{SEM} \pm 0.042)$, whereas it dropped significantly in the stress sessions to 0.083 $\left(\mathrm{SEM} \pm 0.024 ; t_{35}=4.43, p<0.001\right)$. In comparison, the burst index for DG in control sessions was 0.127 (SEM \pm 0.01 ), compared with $0.108(\mathrm{SEM} \pm 0.01)$ in the stress sessions $\left(t_{36}=-1.96\right.$, n.s.). To exclude the possibility that repeated stress exposures influenced firing rates, maximum and average firing rate changes from early recordings were compared with later recordings. No significant differences were found between those groups for any of the sub-regions (CA1: maximum firing rate $t_{20}=0.94$, n.s., average firing rate $t_{20}=0.65$, n.s.; CA3: maximum firing rate $t_{12}=0.18$, n.s., average firing rate $t_{12}=-0.34$, n.s.; DG: maximum firing rate $t_{44}=0.37$, n.s., average firing rate $t_{44}=-0.79$, n.s.). In addition, 96 (CA1: $N=43$; CA3: $N=20$ and DG: $N=33$ ) cells which were sampled in the same recording environment and in two separate sessions, from non-stressed Wistar rats in our database were analyzed for the change of firing characteristics (see Figure 4 for an overview). As expected in non-stressed animals no network-wide change in firing frequency was observed (average firing frequency: CA1: $Z_{42}=-0.39$, n.s.; CA3: $Z_{19}=-1.18$, n.s.; DG: $Z_{32}=-0.31$, n.s.; maximum firing frequency: CA1: $Z_{42}=-0.02$, n.s.; CA3:
$Z_{19}=-1.34$, n.s.; DG: $Z_{32}=-0.71$, n.s.). Although individual neurons may change its firing frequency in one direction in naïve rats no network-wide down- or up-regulation can be observed in constant environmental recording conditions. This is supported by our data from stressed animals were individual neurons go against the trend of the network and show an increased firing pattern (see an example in Figure 3, 11.1 -> 16.3 Hz increase).

A striking characteristic of DG granule cells is that they show, apart from their main spatial firing field, other specific firing locations - so called subfields (Jung and McNaughton, 1993; Leutgeb et al., 2007). Detailed analysis revealed that as expected the main firing fields did not change in their firing characteristics (average firing frequency: $t_{46}=1.23$, n.s.). However, subfields appear to be affected. First, not all subfields ( $\sim 45 \%)$ could be matched between the sessions based on their preferred firing location. Secondly, subfields showed a marked decrease in their average firing frequency in the stress session (all subfields included: $t_{125}=2.10, p=0.037$; matching subfields only: $\left.t_{85}=1.93, p=0.057\right)$.

Spatial firing characteristics were mainly unaffected in cells recorded from stressed animals. The spatial information content 
was unaffected for both the CA1 $\left(Z_{33}=-1.07\right.$, n.s. $)$ and CA3 regions $\left(Z_{17}=-1.35\right.$, n.s. $)$, but showed a significant decrease in DG units $\left(Z_{46}=-2.18, p=0.029\right)$. Spatial selectivity was unaffected in all three regions (CA1: $Z_{33}=-1.17$, n.s.; CA3: $Z_{17}=-0.4$, n.s.; DG: $Z_{46}=-1.64$,n.s.). The size of the place fields was unaffected in CA1 $\left(Z_{33}=-0.98\right.$, n.s. $)$ and DG $\left(Z_{46}=-1.64\right.$, n.s.), but showed a decrease in CA3 $\left(Z_{17}=-3.15, p=0.002\right.$; see Table 1). As a measure of exploration we sought to calculate the number of visited bins between sessions and this criterion did not show any significant difference for the CAl and CA3 sub-regions. However a slight decrease for the DG population was found (CA1: $Z_{33}=-0.63$, n.s.; CA3: $Z_{17}=-1.90$, n.s.; DG: $\left.Z_{46}=-3.04, p=0.002\right)$. The number of passes for each bin showed no significant change for any of the sub-regions (Repeated measure ANOVA: $0-14$ passes/bin which includes $99.9 \%$ of all data DG: $F=-2.42, p=$ n.s.; CA3: $F=4.054$, n.s.; CA1: $F=0.47$, n.s.).

\section{DISCUSSION}

Place cell firing activity decreased significantly in both the CA1 and CA3 subareas of the hippocampus after acute photic stress whereas place cells within the DG generally did not display a change in firing activity within a constant environmental setting. No prominent changes in spatial characteristics of place cells were observed. These results strongly support the notion that the hippocampal network undergoes a generalized subregional decrease in excitability and thus reduced processing capabilities after stress. In addition, CA1 and CA3 place cells also showed a decrease in their burst activity which provides further evidence that neuronal excitability is decreased within the CA1-CA3 population of pyramidal neurons. The decreased size of CA3 place fields during stress conditions appear to be a result of the decrease in neuronal firing which affects the generally bigger place fields of CA3 units in a more profound way than the smaller place fields of CA1 place cells. In contrast, DG cells neither show an overall decreased burst activity nor a general firing frequency decrease. However, the decrease of subfield activity in stress recordings as compared to stable mainfield firing rate is an interesting finding. But as the number of spikes within those subfields normally account for only a fraction of the neuronal discharge it might be the reason why overall firing activity appears to be constant in the DG. The high number of subfields which could not be correlated between sessions might suggest a plasticity effect of those subfields and the respective inputs after stress. The decrease in spatial information index for DG units is apparently a result of a reduced exploration pattern during stress recordings, as the change in path length $(r=0.33, p=0.02)$ or occupation of pixels $(r=0.39, p=0.005)$ does positively correlate with the change observed for spatial information content between sessions. It is worth noting that although we see a general decrease of firing activity, individual neurons can increase their firing activity. This result might reflect different inter-hippocampal networks processing as non-essential and essential information are differently affected during this phase of stress recovery (Schwabe et al., 2010).

Importantly, repeated exposures to photic stress did not affect hippocampal unit activity. This is in accordance with another study from our lab (unpublished observations) which has shown that photic stress reliably activates the HPA axis after repeated exposures without signs of stress adaption. In addition, chronic stress effects can be excluded as rats were never stressed on consecutive days nor were the total number of exposures sufficient

Maximum Firing Frequency
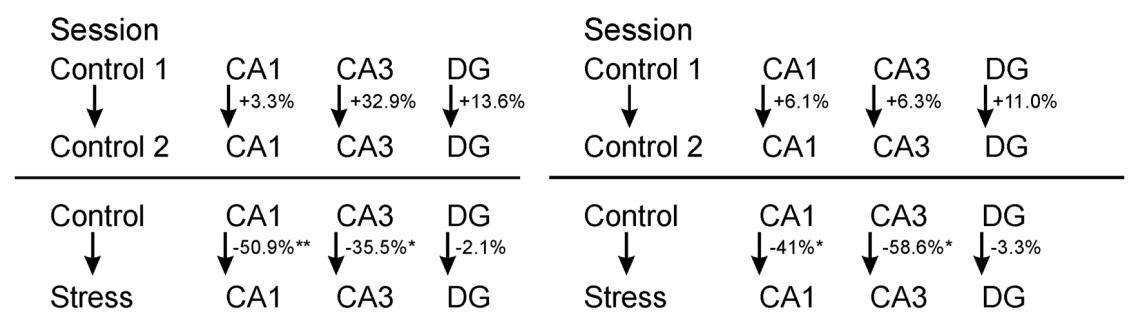

FIGURE 4 | Comparison between neuronal firing frequency changes. Maximum and average firing frequency changes of cells between two consecutive control sessions, as well as one control session and a successive stress session. ${ }^{*} p<0.05,{ }^{* *} p<0.01$.

Table 1 | Main overview of spatial and firing characteristics of all three main sub-regions.

\begin{tabular}{|c|c|c|c|c|c|c|}
\hline & \multicolumn{2}{|c|}{ CA1 } & \multicolumn{2}{|c|}{ CA3 } & \multicolumn{2}{|c|}{ DG } \\
\hline & Control & Stress & Control & Stress & Control & Stress \\
\hline Maximum firing rate $(\mathrm{Hz})$ & $7.64 \pm 0.96^{* *}$ & $5.06 \pm 0.71$ & $13.35 \pm 2.17^{*}$ & $9.85 \pm 1.45$ & $7.37 \pm 1.02$ & $7.21 \pm 1.23$ \\
\hline Average firing rate $(\mathrm{Hz})$ & $0.72 \pm 0.12$ & $0.51 \pm 0.09$ & $1.94 \pm 0.38^{*}$ & $1.22 \pm 0.29$ & $0.90 \pm 0.22$ & $0.87 \pm 0.23$ \\
\hline Size of place field ${ }^{\ddagger}$ & $14.1 \pm 1.97$ & $13.5 \pm 1.36$ & $26.83 \pm 5.14^{*}$ & $21.18 \pm 3.89$ & $15.6 \pm 1.67$ & $16.9 \pm 1.75$ \\
\hline Spatial selectivity & $15.4 \pm 1.61$ & $14.0 \pm 1.41$ & $11.28 \pm 1.91$ & $13.30 \pm 2.53$ & $15.1 \pm 1.51$ & $13.6 \pm 1.69$ \\
\hline Spatial information content ${ }^{\dagger}$ & $1.82 \pm 0.15$ & $1.67 \pm 0.14$ & $1.47 \pm 0.25$ & $1.51 \pm 0.23$ & $1.73 \pm 0.11^{*}$ & $1.53 \pm 0.11$ \\
\hline
\end{tabular}

Values presented as mean values \pm SEM. ${ }^{*} p<0.05,{ }^{*} p<0.01,{ }^{*}$ size of place field expressed in pixels, ${ }^{\dagger}$ Spatial information content in bits per spike. 
to induce chronic stress effects (Joëls et al., 2004). Moreover, the recording procedure per se (control) was neither stressful nor painful for the rats and they were well accustomed to the recording environment before the first stress session.

Our results suggest that firing decreases within area CA1 after stress appear not to be mainly based on rate remapping processes. If stress impairments act mainly via an impairment of rate remapping processes, DG cells are expected to be more profoundly affected, as those cells are regarded as the central mediators of a rate remapping response, experiencing higher changes of firing rates than CA1 and CA3 (Leutgeb et al., 2005, 2007). Hence, the greater stress-induced decrease in firing rate observed in CA1 cells in the study of Kim et al. (2007) during a rate remapping protocol might be a by-product of a systematic CA1/CA3 neuronal "slowdown" response after stress, rather than being specific to rate remapping process as defined by Leutgeb et al. (2007). Nevertheless, the possibility that in processes which require rate remapping, stress has a higher impact under normal firing conditions cannot be excluded as our protocol did not test for those circumstances; we would assume that under more challenging conditions and thus higher hippocampal involvement stress effects would be amplified. In that respect, a study specifically investigating rate or global remapping processes after stress in the DG and/or CA3 and possibly entorhinal cortex would be of further interest. In addition, the difference in the nature of the stressor (audiogenic versus photic), the duration of stress exposure ( $2 \mathrm{~h}$ versus $30 \mathrm{~min}$ ) and the difference in strain of the animals between the two studies might provide some additional explanation.

Our data indicate that acute photic stress alters only CA1 and CA3 place cell activity without an overall affect on the DG granule cells in their firing capacity during freely moving exploration in a constant environment. This observation is an important translation from earlier in vitro and LTP studies (Foy et al., 1987; Shors et al., 1989; Dubrovsky et al., 1993; Bramham et al., 1998;

\section{REFERENCES}

Aggleton, J. P., O’Mara, S. M., Vann, S. D., Wright, N. F., Tsanov, M., and Erichsen, J. T. (2010). Hippocampal-anterior thalamic pathways for memory: uncovering a network of direct and indirect actions. Eur. J. Neurosci. 31, 2292-2307.

Alfarez, D. N., Wiegert, O., Joëls, M., and Krugers, H. J. (2002). Corticosterone and stress reduce synaptic potentiation in mouse hippocampal slices with mild stimulation. Neuroscience 115, 1119-1126.

Bramham, C. R., Southard, T., Ahlers, S. T., and Sarvey, J. M. (1998). Acute cold stress leading to elevated corticosterone neither enhances synaptic efficacy nor impairs LTP in the dentate gyrus of freely moving rats. Brain Res. 789, 245-255.

Brotons-Mas, J. R., Montejo, N., O'Mara, S. M., and Sanchez-Vives, M. V. (2010). Stability of subicular

Joëls et al., 2006), and to our knowledge is the first freely moving experiment studying all of these structures. The MR-GR balance theory proposed by de Kloet et al. (2005) suggests that, in a time-dependent manner, activation of membrane MRs (via increased corticosterone) amplifies the initial stress response in a feed-forward fashion, and at the same time initiates processes allowing the containment of the initial stress reactions via membrane and mainly genomic GR-mediated feedback. Hence, after the early activation period, the normalization period helps to recover from the stressful event, where corticosterone levels slowly decrease and GR activation increase (de Kloet et al., 2005). During this recovery period there is decreased LTP in CA1 and CA3, but no effect on the DG was observed (Joëls and Krugers, 2007). In summary, it thus appears plausible that decreased hippocampal excitability (specifically CA1 and CA3) during the normalization phase, affects a broader range of functional processes including rate remapping. Those decreased functional hippocampal capabilities within this time period then in turn may attenuate a broad range of memory-associated processes where the hippocampus plays a major role (Aggleton et al., 2010). In this respect, assuming the temporal variable remains constant, memory processes and thus behavioral tasks that rely at least partly on the hippocampus will be negatively affected, where the brain is unable to switch rapidly to a hippocampal-independent system to solve the task or at least compensate for the hippocampal deficits. However, this down-regulation of certain networks may be beneficial in order to facilitate the storage of stress-related memories (Joëls and Krugers, 2007). Thus, in future studies it will be crucial to directly link the neuronal changes induced by a stress exposure with the behavioral deficits or enhancements observed in more challenging memory tasks than in pure exploratory tasks.

Grant sponsor: Science Foundation Ireland; Grant number: 8AA G22112.

Dubrovsky, B., Gijsbers, K., Filipini, D., and Birmingham, M. K. (1993). Effects of adrenocortical steroids on long-term potentiation in the limbic system: basic mechanisms and behavioral consequences. Cell. Mol. Neurobiol. 13, 399-414.

Foy, M. R., Stanton, M. E., Levine, S., and Thompson, R. F. (1987). Behavioral stress impairs long-term potentiation in rodent hippocampus. Behav. Neural Biol. 48, 138-149.

Hollup, S. A., Molden, S., Donnett, J. G., Moser, M. B., and Moser, E. I. (2001). Place fields of rat hippocampal pyramidal cells and spatial learning in the watermaze. Eur. J. Neurosci. 13 1197-1208.

Joëls, M., Karst, H., Alfarez, D., Heine, V. M., Qin, Y., van Riel, E., Verkuyl, M., Lucassen, P. J., and Krugers, H. J. (2004). Effects of chronic stress on structure and cell function in rat hippocampus and hypothalamus. Stress 7, 221-231.
Joëls, M., and Krugers, H. J. (2007). LTP after stress: up or down? Neural Plast. 2007, 93202.

Joëls, M., Pu, Z., Wiegert, O., and Krugers, H. J. (2006). Learning under stress: how does it work? Trends Cogn. Sci. (Regul. Ed.) 10, 152-158.

Jung, M. W., and McNaughton, B. L. (1993). Spatial selectivity of unit activity in the hippocampal granular layer. Hippocampus 3, 165-182.

Karst, H. (2000). Corticosteroid actions in hippocampus require DNA binding of glucocorticoid receptor homodimers. Nat. Neurosci. 3 , 977-978.

Karst, H., Berger, S., Turiault, M., Tronche, F., Schütz, G., and Joëls M. (2005). Mineralocorticoid receptors are indispensable for nongenomic modulation of hippocampal glutamate transmission by corticosterone. Proc. Nat. Acad. Sci. USA 102, 19204-19207. 
Kim, J. J, Koo, J. W., Lee, H. J., and Han, J. -S. (2005). Amygdalar inactivation blocks stress-induced impairments in hippocampal long-term potentiation and spatial memory. J. Neurosci. 25, 1532-1539.

Kim, J. J., Lee, H. J., Welday, A. C., Song, E., Cho, J., Sharp, P. E., Jung, M. W., and Blair, H. T. (2007). Stressinduced alterations in hippocampal plasticity, place cells, and spatial memory. Proc. Natl. Acad. Sci. U.S.A. 104, 18297-18302.

Kole, M. H. P., Koolhaas, J. M., Luiten, P. G., and Fuchs, E. (2001). Highvoltage-activated $\mathrm{Ca}^{2+}$ currents and the excitability of pyramidal neurons in the hippocampal CA3 subfield in rats depend on corticosterone and time of day. Neurosci. Let. 307, 53-56.

LeDoux J. E. (1994). Emotion, memory and the brain. [Review]. Sci. AM 270, 32-39.

Leutgeb, J. K., Leutgeb, S., Moser, M.B., and Moser, E. I. (2007). Pattern separation in the dentate gyrus and CA3 of the hippocampus. Science 315, 961-966.

Leutgeb, S., Leutgeb, J. K., Barnes, C. A., Moser, E. I., McNaughton, B. L., and Moser, M.-B. (2005). Independent codes for spatial and episodic memory in hippocampal neuronal ensembles. Science 309, 619-623.

Leutgeb, S., Leutgeb, J. K., Moser, E. I., and Moser, M.-B. (2006). Fast rate coding in hippocampal CA3 cell ensembles. Hippocampus 16, 765-774.

Leutgeb, S., Leutgeb, J. K., Treves, A. Moser, M.-B., and Moser, E. I. (2004). Distinct ensemble codes in hippocampal areas CA3 and CA1. Science 305, 1295-1298.

McDonald, A. J., and Mascagni F. (1997). Projections of the lateral entorhinal cortex to the amygdala: a Phaseolus vulgaris leucoagglutinin study in the rat. Neuroscience 77, 445-459.

McGaugh, J. L. (2000). Memory - a century of consolidation. Science 287, 248-251.

Muller, R. U., and Kubie, J. L. (1987). The effects of changes in the environment on the spatial firing of hippocampal complex-spike cells. J. Neurosci. 7, 1951-1968.

Muller, R. U., Kubie, J. L., and Ranck, J. B. (1987). Spatial firing patterns of hippocampal complex-spike cells in a fixed environment. J. Neurosci. 7, 1935-1950.

O'Keefe, J., and Conway, D. H. (1978). Hippocampal place units in the freely moving rat: why they fire where they fire. Exp. Brain Res. 31, 573-590.

O'Keefe, J., and Dostrovsky, J. (1971). The hippocampus as a spatial map. Preliminary evidence from unit activity in the freely-moving rat. Brain Res. 34, 171-175.

O'Keefe, J., and Nadel, L. (1978). The Hippocampus as a Cognitive Map. Oxford: Oxford University Press.
Paxinos, G., and Watson, C. (1998). The Rat Brain in Stereotaxic Coordinates. New York, NY: Nature Publishing Group.

Roozendaal, B., Sapolsky, R. M., and McGaugh, J. L. (1998). Basolateral amygdala lesions block the disruptive effects of long-term adrenalectomy on spatial memory. Neuroscience $84,453-465$.

Schwabe, L., Wolf, O. T., and Oitzl, M. S. (2010). Memory formation under stress: quantity and quality. Neurosci. Biobehav. Rev. 34, 584-591.

Shors, T. J., Seib, T. B., Levine, S., and Thompson, R. F. (1989). Inescapable versus escapable shock modulates long-term potentiation in the rat hippocampus. Science 244 224-226.

Skaggs, W. E., McNaughton, B. L. Wilson, M. A., and Barnes, C. A. (1996). Theta phase precession in hippocampal neuronal populations and the compression of temporal sequences. Hippocampus 6, 149-172.

Thompson, L. T., and Best, P. J. (1990). Long-term stability of the placefield activity of single units recorded from the dorsal hippocampus of freely behaving rats. Brain Res. 509, 299-308.

Tsanov, M., Chah, E., Wright, N., Vann, S. D., Reilly, R., Erichsen, J. T., Aggleton, J. P., and O’Mara, S. M. (2011). Oscillatory entrainment of thalamic neurons by theta rhythm in freely moving rats. J. Neurophysiol. 105, 4-17.
Van Gemert, N. G., and Joëls, M. (2006). Effect of chronic stress and RU38486 treatment on voltage-dependent calcium currents in rat hippocampal dentate gyrus. J. Neuroendocrinol. 18, 732-741.

Wiegert, O., Joëls, M., and Krugers, H. (2006). Timing is essential for rapid effects of corticosterone on synaptic potentiation in the mouse hippocampus. Learn. Mem. 13, 110-113.

Conflict of Interest Statement: The authors declare that the research was conducted in the absence of any commercial or financial relationships that could be construed as a potential conflict of interest.

Received: 19 August 2011; accepted: 26 September 2011; published online: 17 October 2011.

Citation: Passecker J, Hok V, DellaChiesa A, Chah E and O'Mara SM (2011) Dissociation of dorsal hippocampal regional activation under the influence of stress in freely behaving rats. Front. Behav. Neurosci. 5:66. doi: 10.3389/fnbeh.2011.00066

Copyright () 2011 Passecker, Hok, DellaChiesa, Chah and O'Mara. This is an open-access article subject to a nonexclusive license between the authors and Frontiers Media SA, which permits use, distribution and reproduction in other forums, provided the original authors and source are credited and other Frontiers conditions are complied with. 